EPJ Web of Conferences 47, 06001 (2013)

DOI: $10.1051 /$ epjconf/20134706001

(C) Owned by the authors, published by EDP Sciences, 2013

\title{
Ultracool dwarfs in open clusters and star-forming regions
}

\author{
Nicolas Lodieu ${ }^{1,2, a}$ \\ ${ }^{1}$ Instituto de Astrofísica de Canarias (IAC), Calle Vía Láctea s/n, 38200 La Laguna, Tenerife, \\ Spain \\ ${ }^{2}$ Departamento de Astrofísica, Universidad de La Laguna (ULL), 38205 La Laguna, Tenerife, \\ Spain \\ ${ }^{3}$ Ramón y Cajal fellow at the IAC
}

\begin{abstract}
In this contribution I review the results of the UKIRT Infrared Deep Sky Survey (UKIDSS) Galactic Clusters Survey (GCS) on the Initial Mass Function (IMF) and the current searches for young T dwarfs in open clusters and star-forming regions.
\end{abstract}

\section{MAIN ACHIEVEMENTS OF THE UKIDSS GCS}

\subsection{The UKIDSS GCS}

The UKIRT Infrared Deep Sky Survey [UKIDSS; 1$]^{1}$ is a deep large-scale infrared survey conducted with the UKIRT Wide field CAMera [WFCAM; 2] equipped with five infrared filters [ZYJHK; 3] and located on the summit of Mauna Kea (Hawaii). All data are pipeline-processed at the Cambridge Astronomical Survey Unit (CASU; Irwin et al. in prep) ${ }^{2}$, processed and archived in Edinburgh, and later released to the community through the WFCAM Science Archive [WSA; 4] $]^{3}$. One of its components, the Galactic Clusters Survey (hereafter GCS) imaged 1000 square degrees homogeneously in ten star-forming regions and open clusters down to $0.03-0.01 \mathrm{M}_{\odot}$ (depending on the age and distance of each region) to investigate the universality of the initial mass function (IMF). In addition to the photometry, the latest releases of the GCS provide proper motions measured from the different epochs, with accuracies $\sim 10$ milli-arcsec per year (mas/yr). The typical $100 \%$ completeness values are $J \sim 19.2 \mathrm{mag}$ and $K \sim 18 \mathrm{mag}$, respectively.

\subsection{GCS targets and main results}

Among the ten open clusters and star-forming regions imaged by the GCS, we present here the results on $\sigma$ Ori [5], Upper Scorpius [6-9], IC4665 [10], $\alpha$ Per [11], the Pleiades [12], and Praesepe [13]. We present examples of the coverage of the GCS in $\alpha$ Per, the Pleiades, and Praesepe in Fig. 1. We display the $(Z-J, Z)$ colour-magnitude diagrams for all clusters in Fig. 2 and the vector point diagrams for $\alpha$ Per, the Pleiades, and Praesepe in Fig. 3. The associated mass function are presented

\footnotetext{
ae-mail: nlodieu@iac.es

${ }^{1}$ The survey is described at www.ukidss.org

${ }^{2}$ The CASU WFCAM webpage is at http://apm15.ast.cam.ac.uk/wfcam

${ }^{3} \mathrm{WSA}$ is accessible at http://surveys.roe.ac.uk/wsa
}

This is an Open Access article distributed under the terms of the Creative Commons Attribution License 2.0, which permits unrestricted use, distribution, and reproduction in any medium, provided the original work is properly cited. 

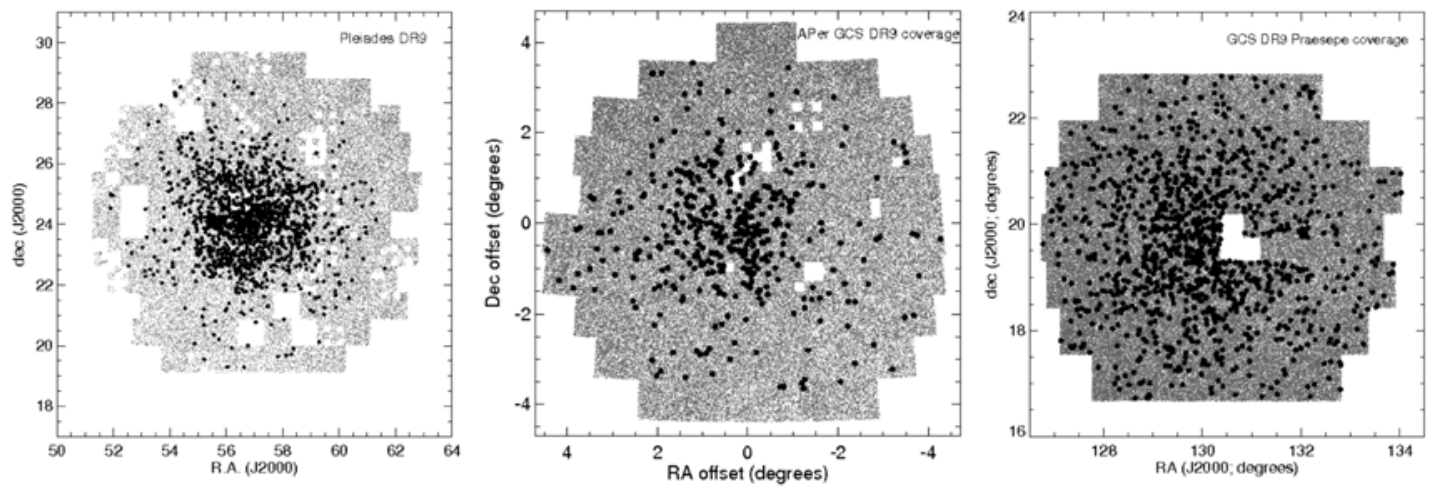

Figure 1. Examples of the UKIDSS GCS DR9 coverage in the $\alpha$ Per $\left(56 \mathrm{deg}^{2}\right.$; left), the Pleiades ( $80 \mathrm{deg}^{2}$; middle), and Praesepe (36 $\mathrm{deg}^{2}$; right) imaged in $Z Y J H K$ with proper motions accurate to a few mas/yr. Overplotted as black dots are cluster member candidates identified in the GCS.
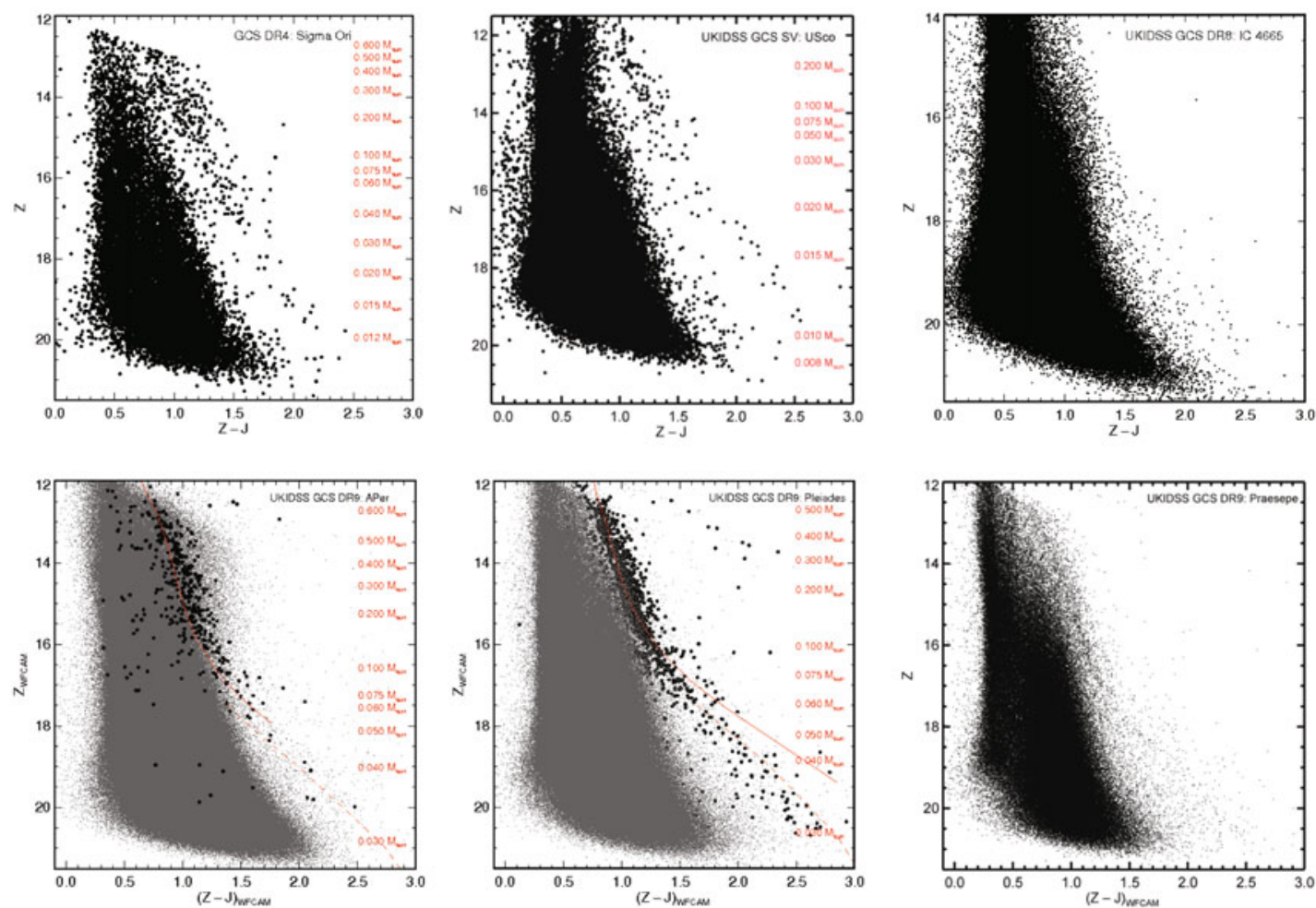

Figure 2. $(Z-J, Z)$ colour-magnitude diagrams for six regions imaged by the UKIDSS GCS (from top to bottom and left to right): $\sigma$ Ori, Upper Scorpius, IC 4665, $\alpha$ Per, the Pleiades, and Praesepe.

in Fig. 4. We detail below our results in each cluster, from the youngest ( $\sim 3-5 \mathrm{Myr})$ to the oldest ( $600 \mathrm{Myr})$ :

- $\sigma$ Orionis is a young (1-8 Myr; [14-16]) cluster located at $450 \mathrm{pc}$ in Orion. We analysed the nearinfrared photometry from the fourth data release of the GCS and recovered most of the previously 


\section{Hot Planets and Cool Stars}
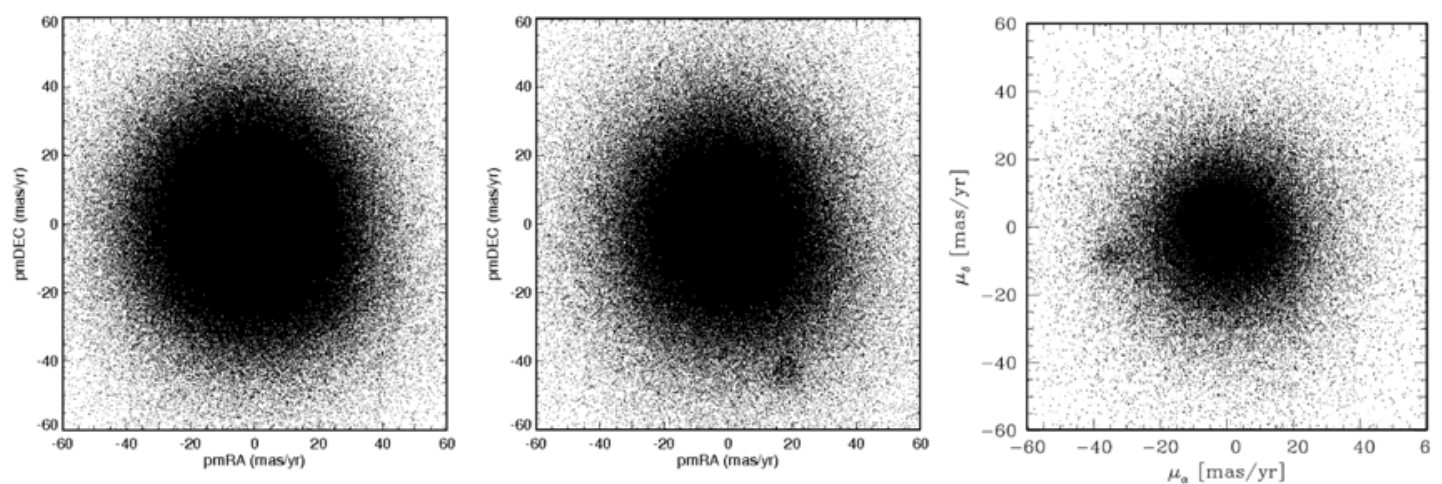

Figure 3. Proper motion vector point diagrams for $\alpha$ Per (left), the Pleiades (middle), and Praesepe (right).
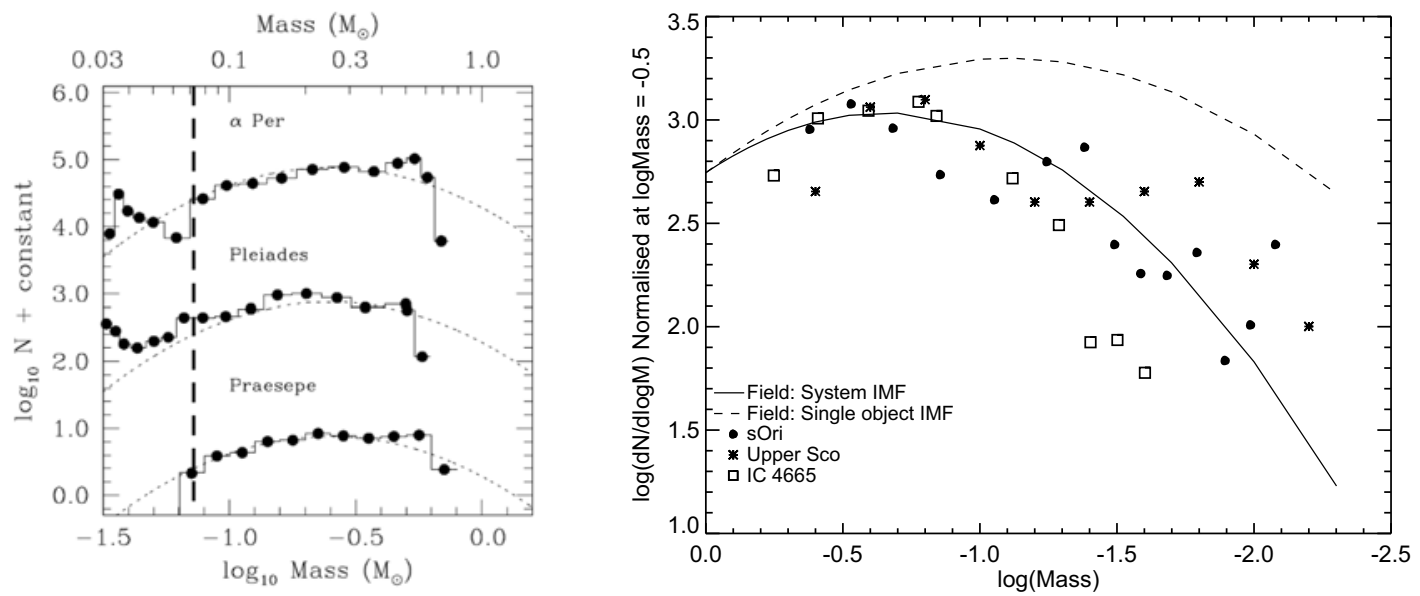

Figure 4. Left: mass functions for $\alpha$ Per, the Pleiades, and Praesepe derived photometrically and astrometrically in an homogeneous manner. The (system) field IMF [33] is overplotted as a dotted line. Right: mass functions for $\sigma$ Ori, Upper Sco, and IC 4665 compared to the lognormal form of the system (solid line) and single (dashed line) field IMF [33].

published members in the central 30 arcmin of the cluster $[17,18]$. We found a total of 287 candidate members down to the deuterium-burning limit with a level of contamination expected below $15 \%$.

- Upper Sco is part of the nearest OB association to the Sun, Scorpius-Centaurus (145 pc, 5-10 Myr; [19-21]). We found that the cluster members define a sequence well separated from field stars in the $(Z-J, Z$ ) colour-magnitude diagram (Fig. 2). We selected a total of 164 candidates with $J=10.5-$ 18.7 mag in 6.5 square degrees surveyed during the science verification phase of the UKIDSS GCS using a variety of colour-magnitude diagrams [7]. We confirmed spectroscopically over $90 \%$ of these photometric candidates as spectroscopic members [9]. We also measured the proper motion of all photometric candidates brighter than $J=15.5$ mag using 2MASS as first epoch $[22,23]$ to further constrain their membership to Upper Sco.

- IC 4665 is a pre-main-sequence cluster with an lithium age of $27 \mathrm{Myr}$ [24] located at $350 \mathrm{pc}$ from the Sun [25]. We cross-matched the near-infrared photometric data from UKIDSS GCS DR8 with optical data from the Canada-France-Hawaii wide-field camera taken a few years earlier [26] to improve the 


\section{EPJ Web of Conferences}

determination of the substellar luminosity and mass functions. We identified 493 bona-fide members from 0.5 down to $0.025 \mathrm{M}_{\odot}$ over 4.3 square degrees common to the optical and infrared surveys. In addition, we extracted new candidate members down to $\sim 0.02 \mathrm{M}_{\odot}$ in a previously unstudied region of the cluster and derived an improved mass function.

- $\alpha$ Per is 80-90 Myr-old [27] and located at a distance of $170 \mathrm{pc}$ from the Sun [28]. The cluster is located at low galactic latitude $\left(b \sim 7^{\circ}\right)$ but shows a significant proper motion, of the order of 35 mas/yr. We exploited the 56 square degree surveyed by GCS DR9 in five passbands (ZYJHK) complemented by proper motions accurate to $\sim 10$ mas $/$ yr. We selected $\sim 700$ cluster member candidates combining photometry and astrometry which we used to derive the luminosity and mass functions. We also provided revised membership for all previously published $\alpha$ Per low-mass stars and brown dwarfs recovered in GCS DR9 based on the new photometry and astrometry [11].

- The Pleiades is the best studied open cluster in the Northern sky because it is fairly young [125 Myr; 29], nearby [120 pc; 28], and has a large mean proper motion ( $~ 50$ mas/yr). We exploited the UKIDSS GCS DR9 in the same manner as for $\alpha$ Per and identified a sample of $\sim 1000$ Pleiades cluster member candidates over 80 square degrees, combining photometry and proper motions derived from the multiple epochs provided by GCS DR9 [12].

- Praesepe is an old (590 Myr; [30]) nearby (182 pc; [28]) cluster with a large mean proper motion of $\sim 38$ mas/yr. As for $\alpha$ Per and the Pleiades, we exploited the GCS DR9 and identified over one thousand photometric and astrometric member candidates in 36 square degrees [13].

\subsection{The mass function}

For all the aforementioned clusters, we derived the luminosity and mass functions using the NextGen and DUSTY models from the Lyon group for consistency $[31,32]$. The range of masses studied by the GCS vary from one region to the other due to the differences in age and distance but, overall, we are sensitive to low-mass stars and brown dwarfs. The mass functions for $\alpha$ Per, the Pleiades, and Praesepe are shown on the left-hand side of Fig. 4 whereas the mass functions for the other regions are displayed on the right-hand of the same figure. We overplotted the log-normal form of the field mass function [33] for comparison. We do not observe any significant difference (at the $3 \sigma$ level) between the shape of all mass functions although some differences are present.

Our main conclusion regarding the universality of the IMF from the homogeneous dataset provided by the UKIDSS GCS is supported by the overall summary of a recent review on the IMF [34]. These authors concluded that (to quote them) "the IMF at the stellar-substellar boundary is still under investigation and uncertainties remain large, but most observations are consistent with a IMF that declines well below the hydrogen-burning limit"; they added that "there is no clear evidence that the IMF varies strongly and systematically as a function of initial conditions".

\section{REVIEW ON SEARCHES FOR YOUNG T DWARFS}

Several young regions and open clusters have been surveyed deeply to uncover genuine young T dwarfs. Below, we summarise each survey, compiled in Figure 5, and provide their main results:

- A large number of $\mathrm{L}$ and $\mathrm{T}$ member candidates were reported in $\rho$ Oph $(\sim 1 \mathrm{Myr})$ from a combined 2MASS $\left(J H K_{S}\right)$ and Spitzer (3.6-8.0 $\left.\mu \mathrm{m}\right)$ survey [35], including a mid-T dwarf from low-resolution near-infrared spectroscopy [42]. If member of the cloud, its mass would be a few Jupiter masses. The membership of this object was refuted by [43] using an independent optical and near-infrared survey so its nature remains to be explained.

- The Serpens cloud is one of the nearest (260-400 pc) young ( $\sim 1 \mathrm{Myr})$ examples of clustered star formation. A dedicated narrow-band methane imaging in the $\mathrm{ON}$ and OFF filters was conducted to 


\begin{tabular}{|c|c|c|c|c|c|c|}
\hline Region & candidates & Imaging & PM & Spectro & Remain & Good \\
\hline Rho Oph & 22 & $\vee$ & $x$ & $x \vee$ & $?$ & $1 ?$ \\
\hline Serpens & 4 & $\vee$ & $x$ & $x$ & $1+1 ?$ & 1 \\
\hline IC348 & 3 & $\vee$ & $\vee$ & $x$ & 1 & $?$ \\
\hline sOri & 3 & $\vee$ & $\vee$ & $x \vee$ & 1 & $?$ \\
\hline USco & 5 & $\vee$ & $\checkmark$ & $x$ & 0 & 0 \\
\hline Pleiades & 7 & $\vee$ & $\vee$ & $x$ & 2 & $?$ \\
\hline Hyades & 2 & $\vee$ & $\checkmark$ & $\checkmark$ & 2 & 2 \\
\hline
\end{tabular}

Figure 5. Table showing the regions searched for young $\mathrm{T}$ dwarfs and the number of candidates. References are: $\rho$ Oph [35], Serpens [36], IC 348 [37], $\sigma$ Orionis [38], Upper Sco [39], the Pleiades [40], and the Hyades [41].

uncover T-type candidates in the heavily embedded core [36]: four candidates were identified after adding near-infrared imaging but two of them were classified as non members from their positions in various colour-magnitude and colour-colour diagrams. The membership of the third candidate remain uncertain whereas the fourth presents all the characteristics of a potential young $\mathrm{T}$ dwarf although neither astrometry nor spectroscopy is currently available.

- IC 348 has an age of 1-3 Myr and is located in Perseus where extinction $\left(\mathrm{A}_{V}\right)$ varies from 2 to 20 mag across the cluster. Three faint $\mathrm{T}$ dwarf candidates were identified in a methane survey combined with infrared photometry [37]. The availability of a deep optical survey rejected two of them as T dwarfs, leaving one potential candidate that remains to be confirmed.

- The deep VISTA survey in $\sigma$ Orionis [38] doubled the number of known planetary-mass objects in the region [44]. The astrometric measurements for the two known T-type candidates [45-47] suggest that they are proper motion non members at the $2 \sigma$ level. One new T candidate was identified (SOri J053804.65-021352.5; [38]) but lacks astrometry and spectroscopy.

- Five T-type candidates were reported in Upper Sco from a deep $Y J$ survey complemented by methane imaging [39]. We obtained new $z$-band imaging with GTC/OSIRIS, suggesting that their optical-toinfrared colours are inconsistent with field T dwarfs. Additionally, new $H$-band photometry taken with ESO NTT/SofI four years after the deep $Y J$ survey yields proper motions inconsistent with the mean motion of the association at $>2 \sigma$ (Lodieu et al. 2013, subm. to MNRAS).

- Several photometric L and T dwarf member candidates were identified in the Pleiades [40] thanks to the large baseline between the optical CFHT survey [48] and the GCS [49], yielding proper motions for the faintest sources using both surveys. However, up to now, none of them has been confirmed as a bona-fide member from complementary methane imaging and Spitzer mid-infrared photometry [50]. Spectroscopy is hard but needed to assess their nature.

- Two early-T dwarfs were announced in the Hyades [41]. These unambiguous 650 Myr-old T1 and $\mathrm{T} 2$ dwarfs with a mass of $0.05 \mathrm{M}_{\odot}$ were confirmed via astrometry (i.e. two epoch photometry) and low-resolution near-infrared spectroscopy after their original discovery in 16 square degrees surveyed in $i, z$ with complementary $K$-band photometry.

To sum up, no bona-fide young $\mathrm{T}$ dwarfs $(<150 \mathrm{Myr})$ have been confirmed photometrically, astrometrically, and spectroscopically as of today. Only two T dwarfs have been unambigously identified in the Hyades [41]. Many independent searches are currently on-going, promising the discovery of the first young methane brown dwarf(s) in the coming years. 
EPJ Web of Conferences

\section{CONCLUSIONS}

We would like to conclude this review with two messages to take home: firstly, the shape of the IMF in the low-mass and substellar regimes from the homogeneous survey of young star-forming regions and open clusters conducted as part of the GCS does not show any significant variation, and, secondly, no unambiguous T-type dwarf has been confirmed as of today in regions younger than $150 \mathrm{Myr}$ despite the numerous searches conducted over the past years.

NL was funded by the Spanish Ministry of Economics and Competitiveness (project AYA2010-19136), the Ramón y Cajal fellowship program, and RoPACS. I would like to thank my collaborators (with a special thank to Nigel Hambly for designing the GCS and his support throughout the years) as well as the UKIDSS, CASU and Edinburgh teams for all their work and support.

\section{References}

[1] A. Lawrence, S.J. Warren, O. Almaini, A.C. Edge, N.C. Hambly, 17 co-authors, MNRAS 379, 1599 (2007), arXiv:astro-ph/0604426

[2] M. Casali, A. Adamson, C. Alves de Oliveira, O. Almaini, K. Burch, T. Chuter, J. Elliot, 23 co-authors, A\&A 467, 777 (2007)

[3] P.C. Hewett, S.J. Warren, S.K. Leggett, S.T. Hodgkin, MNRAS 367, 454 (2006)

[4] N.C. Hambly, R.S. Collins, N.J.G. Cross, R.G. Mann, M.A. Read, E.T.W. Sutorius, I. Bond, J. Bryant, J.P. Emerson, A. Lawrence et al., MNRAS 384, 637 (2008), arXiv: 0711.3593

[5] N. Lodieu, M.R. Zapatero Osorio, R. Rebolo, E.L. Martín, N.C. Hambly, A\&A 505, 1115 (2009), 0907.2185

[6] N. Lodieu, N.C. Hambly, R.F. Jameson, MNRAS 373, 95 (2006)

[7] N. Lodieu, N.C. Hambly, R.F. Jameson, S.T. Hodgkin, G. Carraro, T.R. Kendall, MNRAS 374, 372 (2007), astro-ph/0610140

[8] N. Lodieu, N.C. Hambly, R.F. Jameson, S.T. Hodgkin, MNRAS 383, 1385 (2008), arXiv:0711.1109

[9] N. Lodieu, P.D. Dobbie, N.C. Hambly, A\&A 527, A24 (2011), 1101.0919

[10] N. Lodieu, W.J. de Wit, G. Carraro, E. Moraux, J. Bouvier, N.C. Hambly, A\&A 532, A103 (2011), 1106.3957

[11] N. Lodieu, N.R. Deacon, N.C. Hambly, S. Boudreault, MNRAS 426, 3403 (2012), 1207.6978

[12] N. Lodieu, N.R. Deacon, N.C. Hambly, MNRAS p. 2699 (2012)

[13] S. Boudreault, N. Lodieu, N.R. Deacon, N.C. Hambly, MNRAS 426, 3419 (2012), 1208.0466

[14] J.M. Oliveira, R.D. Jeffries, M.J. Kenyon, S.A. Thompson, T. Naylor, A\&A 382, L22 (2002)

[15] M.R. Zapatero Osorio, V.J.S. Béjar, E.L. Martín, D. Barrado y Navascués, R. Rebolo, ApJL 569, L99 (2002)

[16] W.H. Sherry, F.M. Walter, S.J. Wolk, AJ 128, 2316 (2004), arXiv : astro-ph/0410244

[17] J.A. Caballero, A\&A 478, 667 (2008), arXiv: 0710.5882

[18] V.J.S. Béjar, M.R. Zapatero Osorio, R. Rebolo, J.A. Caballero, D. Barrado, E.L. Martín, R. Mundt, C.A.L. Bailer-Jones, ApJ 743, 64 (2011), 1109. 1210

[19] J.H.J. de Bruijne, R. Hoogerwerf, A.G.A. Brown, L.A. Aguilar, P.T. de Zeeuw, Improved Methods for Identifying Moving Groups, in ESA SP-402: Hipparcos - Venice '97 (1997), pp. 575-578

[20] T. Preibisch, H. Zinnecker, AJ 123, 1613 (2002)

[21] M.J. Pecaut, E.E. Mamajek, E.J. Bubar, ApJ 746, 154 (2012), 1112.1695

[22] R.M. Cutri, M.F. Skrutskie, S. van Dyk, C.A. Beichman, J.M. Carpenter, T. Chester, L. Cambresy, T. Evans, J. Fowler, J. Gizis et al., 2MASS All Sky Catalog of point sources 2246 (2003) 


\section{Hot Planets and Cool Stars}

[23] M.F. Skrutskie, R.M. Cutri, R. Stiening, M.D. Weinberg, S. Schneider, J.M. Carpenter, 25 coauthors, AJ 131, 1163 (2006)

[24] S. Manzi, S. Randich, W.J. de Wit, F. Palla, A\&A 479, 141 (2008), arXiv: 0712.0226

[25] N.V. Kharchenko, A.E. Piskunov, S. Röser, E. Schilbach, R.D. Scholz, A\&A 438, 1163 (2005), arXiv: astro-ph/0501674

[26] W.J. de Wit, J. Bouvier, F. Palla, J.C. Cuillandre, D.J. James, T.R. Kendall, N. Lodieu, M.J. McCaughrean, E. Moraux, S. Randich et al., A\&A 448, 189 (2006), astro-ph/0511175

[27] D. Barrado y Navascués, J.R. Stauffer, R. Jayawardhana, ApJ 614, 386 (2004)

[28] F. van Leeuwen, A\&A 497, 209 (2009), 0902.1039

[29] J.R. Stauffer, G. Schultz, J.D. Kirkpatrick, ApJL 499, 219 (1998)

[30] L. Fossati, S. Bagnulo, J. Landstreet, G. Wade, O. Kochukhov, R. Monier, W. Weiss, M. Gebran, A\&A 483, 891 (2008), 0803.3540

[31] I. Baraffe, G. Chabrier, F. Allard, P.H. Hauschildt, A\&A 337, 403 (1998)

[32] G. Chabrier, I. Baraffe, F. Allard, P. Hauschildt, ApJ 542, 464 (2000)

[33] G. Chabrier, The Initial Mass Function: from Salpeter 1955 to 2005, in The Initial Mass Function 50 Years Later, edited by E. Corbelli, F. Palla, \& H. Zinnecker (2005), Vol. 327 of Astrophysics and Space Science Library, p. 41, arXiv : astro-ph/0409465

[34] N. Bastian, K.R. Covey, M.R. Meyer, ARA\&A 48, 339 (2010), 1001.2965

[35] K.A. Marsh, J.D. Kirkpatrick, P. Plavchan, ApJL 709, L158 (2010), 0912.3774

[36] L. Spezzi, G. de Marchi, N. Panagia, A. Sicilia-Aguilar, B. Ercolano, MNRAS 421, 78 (2012), 1111.0835

[37] A.S.M. Burgess, E. Moraux, J. Bouvier, C. Marmo, L. Albert, H. Bouy, A\&A 508, 823 (2009), 0909.0917

[38] K. Peña Ramírez, V.J.S. Béjar, M.R. Zapatero Osorio, M.G. Petr-Gotzens, E.L. Martín, ApJ 754, 30 (2012), 1205.4950

[39] N. Lodieu, N.C. Hambly, P.D. Dobbie, N.J.G. Cross, L. Christensen, E.L. Martin, L. Valdivielso, MNRAS 418, 2604 (2011), 1108.4783

[40] S.L. Casewell, P.D. Dobbie, S.T. Hodgkin, E. Moraux, R.F. Jameson, N.C. Hambly, J. Irwin, N. Lodieu, MNRAS 378, 1131 (2007), arXiv: 0704 . 1578

[41] J. Bouvier, T. Kendall, G. Meeus, L. Testi, E. Moraux, J.R. Stauffer, D. James, J.C. Cuillandre, J. Irwin, M.J. McCaughrean et al., A\&A 481, 661 (2008), 0801.0670

[42] K.A. Marsh, P. Plavchan, J.D. Kirkpatrick, P.J. Lowrance, R.M. Cutri, T. Velusamy, ApJ 719, 550 (2010), 1006.2506

[43] C. Alves de Oliveira, E. Moraux, J. Bouvier, H. Bouy, C. Marmo, L. Albert, A\&A 515, A75 (2010), 1003.2205

[44] M.R. Zapatero Osorio, V.J.S. Béjar, E.L. Martín, R. Rebolo, D. Barrado y Navascués, C.A.L. Bailer-Jones, R. Mundt, Science 290, 103 (2000)

[45] M.R. Zapatero Osorio, V.J.S. Béjar, E.L. Martín, R. Rebolo, D. Barrado y Navascués, R. Mundt, J. Eislöffel, J.A. Caballero, ApJ 578, 536 (2002)

[46] G. Bihain, R. Rebolo, M.R. Zapatero Osorio, V.J.S. Béjar, I. Villó-Pérez, A. Díaz-Sánchez, A. Pérez-Garrido, 9 co-authors, A\&A 506, 1169 (2009), 0909.0802

[47] K. Peña Ramírez, M.R. Zapatero Osorio, V.J.S. Béjar, R. Rebolo, G. Bihain, A\&A 532, A42 (2011), 1105.4043

[48] E. Moraux, J. Bouvier, J.R. Stauffer, A\&A 367, 211 (2001)

[49] N. Lodieu, P.D. Dobbie, N.R. Deacon, S.T. Hodgkin, N.C. Hambly, R.F. Jameson, MNRAS 380, 712 (2007), arXiv:0706.2234

[50] S.L. Casewell, R.F. Jameson, M.R. Burleigh, P.D. Dobbie, M. Roy, S.T. Hodgkin, E. Moraux, MNRAS 412, 2071 (2011), 1011.4218 